\title{
International Reports of Unexpected Low Plasma Concentrations of Dabigatran Suggest That More Frequent Measurements Will Add Value
}

\author{
Ruth L. Savage, MBBS, MSc (Clin Pharmacol) 1,2,3 Marilina Castellano, MPharm \\ Michael V. Tatley, MBChB, FFCH(SA), FAFPHM, FNZCPHM, BBusSci (Hon) ${ }^{2}$
}

\author{
${ }^{1}$ WHO Collaborating Centre for International Drug Monitoring, \\ Uppsala Monitoring Centre, Uppsala, Sweden \\ ${ }^{2}$ New Zealand Pharmacovigilance Centre, Department of \\ Preventive and Social Medicine, University of Otago, \\ Dunedin, New Zealand \\ ${ }^{3}$ Department of General Practice, University of Otago, Christchurch, \\ New Zealand
}

Semin Thromb Hemost 2017;43:635-638
Address for correspondence Ruth L. Savage, MBBS, MSC (Clin Pharmacol), Centre for Adverse Reactions Monitoring (CARM), New Zealand Pharmacovigilance Centre, Department of Preventive and Social Medicine, University of Otago, PO Box 913, Dunedin, New Zealand (e-mail: ruth.savage@otago.ac.nz).
We were interested to read the report by Chin ${ }^{1}$ recently published in this journal, suggesting that some patients may benefit from dose adjustment of non-vitamin $\mathrm{K}$ antagonist oral anticoagulants (NOACs), otherwise referred to as direct oral anticoagulants (DOACs). We would like to report a small case series that provides evidence for selective dose adjustment and/or measurement of NOACs, in particular dabigatran.

The World Health Organization (WHO) Global Database of Individual Case Safety Reports ("VigiBase") holds reports of suspected adverse drug reactions collected worldwide through the WHO Program for International Drug Monitoring. ${ }^{2}$ A previous investigation of reports of thromboembolic events associated with dabigatran in VigiBase ${ }^{3}$ included a literature search, which identified four published case reports of dabigatran plasma concentrations below the expected "within therapy range" (representing the generally reported range of values identified in patients on such therapy in clinical trials). VigiBase was therefore searched for similar reports of low plasma concentrations. It is acknowledged, however, that the information provided in VigiBase is heterogeneous (i.e., it originates from multiple sources [different countries and types of reporters] and the amount of information given, as well as the likelihood that the medicine caused the adverse reaction, may vary from case to case). Two of the published reports and 12 additional reports of this unexpected effect were identified, originating from five countries.

While specific target therapeutic ranges for dabigatran plasma concentrations have not been validated, "within therapy" ranges identifying values that are typically seen in treated patients have been described. Thus, "expected" trough and peak dabigatran plasma levels after intake of approved dosages for atrial fibrillation are around 61 to 143 and 117 to $275 \mathrm{ng} / \mathrm{mL}$, respectively, for $150 \mathrm{mg}$ twice a day and 43 to 102 and 85 to $200 \mathrm{ng} / \mathrm{mL}$, respectively, for $110 \mathrm{mg}$ twice a day. ${ }^{4}$ Chin calculated an optimal trough range of 30 to $130 \mathrm{ng} / \mathrm{mL}$ after constructing a combined risk versus trough concentration model for a typical patient based on data from the RE-LY (Randomized Evaluation of Long-term anticoagulant therapY) trial. ${ }^{1}$

- Table 1 shows the details of the four published case reports. Patients 1 and 2 had embolic stroke while taking dabigatran. ${ }^{5,6}$ Peak and trough plasma concentrations were measured thereafter using Hemoclot, a commercially available dilute thrombin time (dTT) assay, following witnessed intake. Patients 3 and 4 did not have thromboembolic events. ${ }^{7,8}$ Patient 3 had a lower than expected activated partial thromboplastin time (aPTT) shortly after starting dabigatran, so that peak concentrations at two dose levels were quantified, using Hemoclot, following confirmed intake after 31 days of treatment. Patient 4, who had undergone gastric bypass surgery, had trough concentrations quantified because another patient with gastric bypass developed a cardioembolic stroke on dabigatran and was found to have lower than expected aPTT values. The doses of dabigatran administered were appropriate for all four patients, especially after the dose increase in patient 3 who had normal published online June 13, 2017
Issue Theme Editorial Compilation IV; Guest Editors: Emmanuel J. Favaloro, PhD, FFSc (RCPA), and Giuseppe Lippi, MD.
Copyright @ 2017 by Thieme Medical Publishers, Inc., 333 Seventh Avenue, New York, NY 10001, USA.

Tel: +1(212) 584-4662.
DOI https://doi.org/ 10.1055/s-0037-1603361. ISSN 0094-6176. 


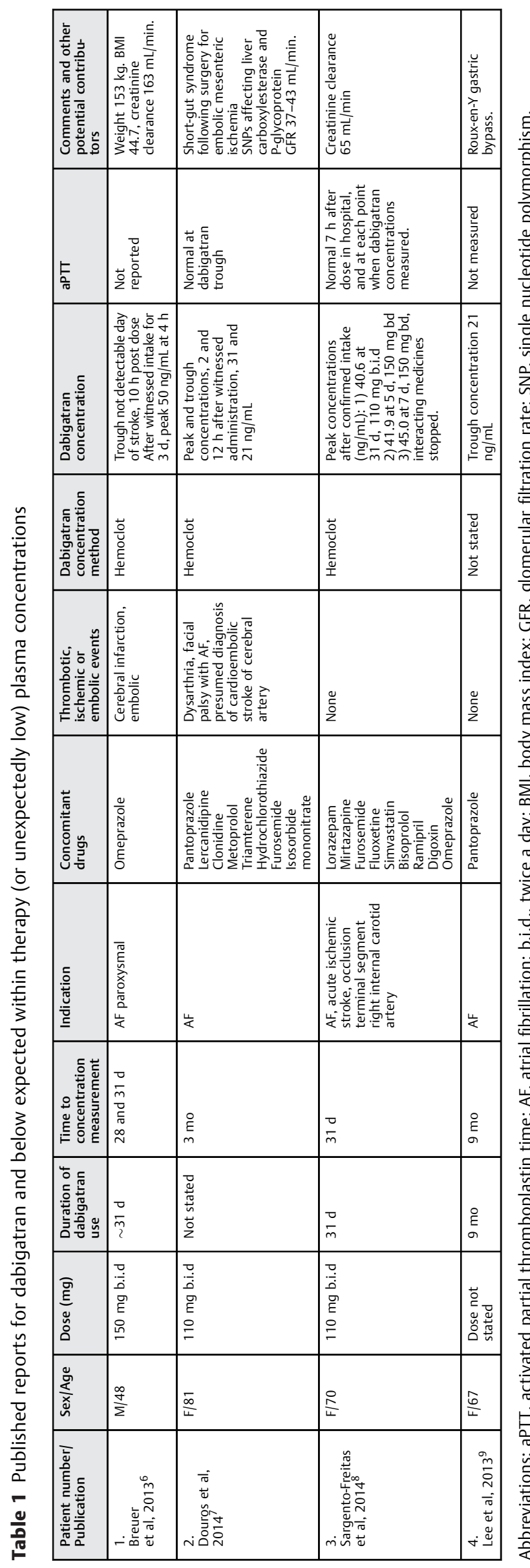

renal function. The most likely contributors to concentrations below the within therapy range were obesity with glomerular hyperfiltration in patient 1 and major gastrointestinal surgery in patients 2 and 4 .

The documentation provided in the 12 additional reports that were not published is more limited. Nevertheless, together they add weight to the findings in the published reports and convey physicians' concerns.

- Table 2 summarizes the 16 reports, published and unpublished. dTT assays were used to quantify plasma dabigatran in 12 reports. The method used was not stated in the remaining four. Two of these reported a concentration and the other two indicated that dabigatran was not detectable.

Eight of the patients had cerebral or venous thromboembolic events, occurring between 3 and 270 days after starting dabigatran. Dabigatran concentrations were below the within therapy range or were too low to be quantified on the same day or shortly after the events while the patients were still taking dabigatran, two (-Table 1, patients 1 and 2) after witnessed administration. Of the patients who did not experience thromboembolic events, low concentrations were suspected and confirmed in two (-Table 1, patients 3 and 4 ). Three patients were found to have normal or lower than expected aPTTs, prior to cardioversion. Using a dTT method, dabigatran concentrations were found to be well below the expected within therapy trough level, with little difference when one patient continued dabigatran for a further 21 days. There was serious concern about cardioversion being delayed. The remaining three reports were submitted because each patient had undetectable peak and trough dabigatran concentrations measured by Hemoclot at 2 and 12 hours after witnessed intake. Duration of dabigatran use and the reason for measurement were not stated.

Overall dabigatran concentrations ( - Table 2 ) were undetectable or below the expected trough range except for one peak level that was within the trough range at $50 \mathrm{ng} / \mathrm{mL}$.

Renal function is important in this context if there is glomerular hyperfiltration, as seen in - Table $\mathbf{1}$ (patient 1 ) or if a lower dose is used when renal function is normal. Four patients received the lower dose of $110 \mathrm{mg}$ twice a day. One had a glomerular filtration rate of 37 to $43 \mathrm{~mL} / \mathrm{min}$ and one (-Table 1, patient 3 ) did not achieve expected within therapy dabigatran concentrations when her dose was increased. Underdosing was a potential cause of low concentrations in the other two patients as their renal function was not recorded.

- Table 2 shows the potential single and multiple contributors to subtherapeutic dabigatran concentrations. In six reports there were no apparent contributors.

Modeling of the data from the RE-LY trial by Chin et al led them to question the conclusion that dabigatran has a wide therapeutic index. ${ }^{9}$ They also asked whether the characteristics of patients with trough concentrations falling below or above the 10th and 90th percentiles could be described. Our case series suggests potentially relevant characteristics occurring alone or in combination for patients with very low measured concentrations. 
Table 2 Characteristics of patients in 16 published and VigiBase reports of below expected within therapy dabigatran plasma concentrations

\begin{tabular}{|c|c|c|c|}
\hline \multicolumn{2}{|l|}{ Characteristics (no. of patients with information) } & \multirow{2}{*}{\multicolumn{2}{|c|}{$\begin{array}{l}\text { Number or range } \\
9 / 6\end{array}$}} \\
\hline Sex (15) & Males/females & & \\
\hline Age $(y)(12)$ & Median (range) & \multicolumn{2}{|c|}{$65.5(48-81)$} \\
\hline \multirow[t]{3}{*}{ Indication (12) } & AF/flutter & \multicolumn{2}{|l|}{10} \\
\hline & AF and DVT & \multicolumn{2}{|l|}{1} \\
\hline & DVT/PE & \multicolumn{2}{|l|}{1} \\
\hline Daily dose (12) & $150 \mathrm{mg} \mathrm{bd} / 110 \mathrm{mg}$ b.i.d & \multicolumn{2}{|l|}{$8 / 4$} \\
\hline Time from dabigatran start to measurement (d) (10) & Median (range) & \multicolumn{2}{|c|}{$36.5(16-270)$} \\
\hline \multirow[t]{3}{*}{ Thrombotic/embolic/ischemic events (8) } & CVA (one with ventricular clot) & \multicolumn{2}{|l|}{4} \\
\hline & TIA & \multicolumn{2}{|l|}{1} \\
\hline & DVT/PE & \multicolumn{2}{|l|}{3} \\
\hline Compliance recorded (6) & Witnessed intake ${ }^{a}$ & \multicolumn{2}{|l|}{6} \\
\hline \multicolumn{4}{|l|}{ Potential contributors to low plasma concentrations } \\
\hline \multirow[t]{3}{*}{ Weight (4) } & BMI obese $(44.7,35.6)$ & \multicolumn{2}{|l|}{2} \\
\hline & Obese & \multicolumn{2}{|l|}{1} \\
\hline & Weight $>100 \mathrm{~kg}$ & \multicolumn{2}{|l|}{1} \\
\hline \multirow[t]{2}{*}{ Major GI surgery (3) } & Short-gut syndrome & \multicolumn{2}{|l|}{1} \\
\hline & Gastric bypass surgery & \multicolumn{2}{|l|}{2} \\
\hline \multirow[t]{2}{*}{ Potentially interacting medicines (8) } & PPIs & \multicolumn{2}{|l|}{7} \\
\hline & Atorvastatin & \multicolumn{2}{|l|}{1} \\
\hline \multirow[t]{5}{*}{$\begin{array}{l}\text { Patients with multiple potential contributors to low dabigatran } \\
\text { concentrations (5) }\end{array}$} & $\begin{array}{l}\text { Short-gut syndrome, genotypes, } \\
\text { PPI }\end{array}$ & \multicolumn{2}{|l|}{1} \\
\hline & $\begin{array}{l}\text { BMI 44.7, high creatinine clear- } \\
\text { ance, PPI }\end{array}$ & \multicolumn{2}{|l|}{1} \\
\hline & Atorvastatin, PPI & \multicolumn{2}{|l|}{1} \\
\hline & Weight $110 \mathrm{~kg}, \mathrm{PPI}$ & \multicolumn{2}{|l|}{1} \\
\hline & Gastric bypass, PPI & 1 & \\
\hline No. with no recorded potential contributors to low dabigatran $c$ & ncentrations & 6 & \\
\hline Laboratory measurements & & & \\
\hline Lower than expected coagulation parameters (6) & aPTT & 6 & \\
\hline Dabigatran measurement method (12) & dTT assays (Hemoclot 9) & 12 & \\
\hline Dabigatran plasma concentrations (ng/mL) (16) & Peak and trough (5) & Trough & $0-21$ \\
\hline & & Peak & $0-50$ \\
\hline & Peak alone (1) & 40.6 and & 1.9 \\
\hline & Trough alone (3) & $0-21$ & \\
\hline & Peak or trough not stated (7) & $0-43$ & \\
\hline
\end{tabular}

Abbreviations: aPTT, activated partial thromboplastin time; BMI, body mass index; CVA, cerebrovascular accident; dTT, dilute thrombin time; DVT, deep vein thrombosis; GI, gastrointestinal; PPI, proton pump inhibitor; PE, pulmonary embolism; TIA, transient ischemic attack.

${ }^{a}$ Concentrations measured at 2 and 12 hours post dose (four patients); after 3 days concentration measured 2 hourly for peak level (one patient); peak levels at $110 \mathrm{mg}$ bd, 31 days and $150 \mathrm{mg}$ bd, 5 days (one patient).

Pantoprazole and atorvastatin have each been shown to reduce dabigatran exposure by approximately $20 \%{ }^{1}$ However, the high proportion of proton pump inhibitor (PPI) users in this case series may reflect background use. Dabiga- tran etexilate has low oral bioavailability. Genetic variants decreasing conversion of dabigatran etexilate to active dabigatran by liver carboxylesterase and influencing intestinal p-glycoprotein activity may account for a $15 \%$ decrease in 
dabigatran bioavailability ( Table 1, patient 2 ). ${ }^{6}$ Decrease in gastric acidity due to the use of PPIs and major upper gastrointestinal disorders may also contribute to reduced absorption. Body weight greater than $100 \mathrm{~kg}$ influenced trough levels in the RE-LY study. Because dabigatran is predominantly excreted by the kidneys, glomerular hyperfiltration associated with severe obesity may contribute to low plasma concentrations. ${ }^{5}$

Though it is appropriate to tailor dose to patient characteristics where possible, our series of case reports supports the need for trials in which dabigatran concentrations are measured at the time of thrombotic events, ideally followed by testing after witnessed intake where measured concentrations are low. Investigation is needed into the potential pathways leading to very low plasma concentrations including a combined effect of multiple covariates.

Our data support Chin's suggestion that thrombin inhibition should be appropriately measured more frequently than currently recommended, especially after dabigatran is initiated, after a dose change and when there are changing patient characteristics. The frequency would still be considerably less than that required for vitamin $\mathrm{K}$ antagonist monitoring. ${ }^{1}$

Anticoagulants continue to be one of the major causes of drug-related serious morbidity and death. The lower risk of cerebral bleeding with dabigatran compared with warfarin and its equivalence in other respects is encouraging. By identifying patients who may need finer dose adjustments or do not respond well to dabigatran, and elucidating the causes, we have an even greater opportunity to achieve badly needed improvements in outcomes with only a small loss of convenience regarding testing.

\section{Acknowledgments}

The authors are indebted to the National Pharmacovigilance Centres that contribute data to VigiBase. The opinions and conclusions in this study are not necessarily those of the various centers or of WHO.

\section{References}

1 Chin PKL. Which patients may benefit from dose adjustment of non-vitamin K antagonist oral anticoagulants? Semin Thromb Hemost 2015;41(02):195-207

2 Lindquist M. VigiBase, the WHO Global ICSR Database System: basic facts. Drug Inf J 2008;42:409-419

3 Castellano M, Sartori D, Savage R. Dabigatran and thromboembolism: analysis of case histories for risk factors. WHO Pharmaceuticals Newletter 2016;3:15-22

4 Boehringer Ingelheim. Management of bleeding in emergency situations for patients treated with Pradaxa (dabigatran etexilate). January 2015. Available at: http://products.boehringer-ingelheim.com/pradaxa/sites/default/files/Bleeding_management.pdf. Accessed February 17, 2017

5 Breuer L, Ringwald J, Schwab S, Köhrmann M. Ischemic stroke in an obese patient receiving dabigatran [letter]. N Engl J Med 2013; 368(25):2440-2442

6 Douros A, Schlemm L, Bolbrinker J, Ebinger M, Kreutz R. Insufficient anticoagulation with dabigatran in a patient with short bowel syndrome [letter]. Thromb Haemost 2014;112(02):419-420

7 Sargento-Freitas J, Silva F, Pego J, Duque C, Cordeiro G, Cunha L. Cardioembolic stroke in a patient taking dabigatran etexilate: the first case report of clinical and pharmacologic resistance [letter]. J Neurol Sci 2014;346(1-2):348-349

8 Lee D, DeFilipp Z, Judson K, Kennedy M. Subtherapeutic anticoagulation with dabigatran following Roux-en-Y bypass surgery [letter]. J Cardiol Cases 2013;8:e49-e50

9 Chin PKL, Wright DFB, Doogue MP, Begg EJ. Learning more from the dabigatran concentrations in the RE-LY study. J Am Coll Cardiol 2014;63(24):2746-2747 